\title{
Increasing Social Media Involvement around the American Society of Neuroradiology Annual Meeting
}

I read with great interest the social media vignette by D'Anna et $\mathrm{al}^{1}{ }^{1}$ which describes the analysis of Twitter use during the American Society of Neuroradiology (ASNR) 2018 Annual Meeting. Although the analysis showed a substantial increase in Twitter use during the past 4 years, there is still room for improvement. More robust community engagement can be achieved by implementing some simple-but-practical strategies before the conference even begins.

Submission and acceptance of abstracts for original research articles, case reports, and educational posters are an integral part of every annual meeting and actively involve a large number of physicians. After the announcement of acceptances, development of a method to systematically share the abstract on social media would help increase the radiology community involvement in the conference and social media because physicians from multiple disciplines follow each other across social media platforms. Developing a central hashtag to share these abstracts will further help increase visibility and outreach (eg, \#ASNR20Abstract).
Because a large number of fascinating keynotes, panels, and discussions take place during the conference, it is understandable that social media visibility for different events will vary because it depends on the social media involvement of the main speaker. However, to overcome this issue, the main Twitter account of the conference can conduct various Tweet chats beginning 2-3 months before the conference, and multiple speakers can give brief introductions of their topics. Conducting these Tweet chats will help increase the visibility of these events and generate enthusiasm for the actual event.

\section{REFERENCE}

1. D'Anna G, Chen MM, McCarty JL, et al. The continued rise in professional use of social media at scientific meetings: an analysis of Twitter use during the ASNR 2018 annual meeting. AJNR Am J Neuroradiol 2019;40:935-97 CrossRef Medline

(iD) T. Garg Seth GS Medical College and KEM Hospital Mumbai, Maharashtra, India 